\title{
Les lipides polaires : actifs et vecteurs cosmétiques
}

Agnès LEFUR

Jean-Pierre ARNAUD

Lucas Meyer Cosmetics,

99 , route de Versailles,

91160 Champlan, France

Les lipides polaires constituent une classe à part dans les matières grasses puisque comme leur nom l'indique, ces lipides possèdent une partie hydrophile qui leur permet de jouer un rôle prépondérant au niveau des interfaces que ce soit dans les organismes vivants ou dans les systèmes dispersés.

Ces lipides polaires sont également connus par certaines de leurs sous-classes telles que les lécithines, phospholipides, glycolipides et autres structures plus ou moins complexes. Ces composés ont fait l'objet d'études importantes tant au niveau de la biologie, de la médecine, de l'alimentaire et dans le domaine qui nous intéresse c'est-à-dire la cosmétique. En effet, il a été découvert il y a plus d'un siècle que ces lipides polaires constituent la membrane cellulaire de tout organisme vivant et que par conséquent ils influencent tous les processus métaboliques. Plus proche de nous, l'industrie alimentaire les utilise abondamment dans des préparations aussi recherchées que le chocolat, la baguette française ou les poudres de lait instantanées mettant à profit leurs propriétés mouillantes ou émulsionnantes. L'industrie cosmétique tire d'ailleurs partie de celles-ci dans les produits de maquillage pour disperser efficacement les pigments. Plus récemment, les produits de soin utilisent ces lipides membranaires comme émulsionnant parfaitement toléré et que le marketing revendique quelquefois sous le terme de biocompatible.

Grâce à leur origine membranaire, la diététique utilise les phospholipides comme complément nutritionnel pour protéger nos membranes dans la lutte contre le vieillissement. La cosmétique en fait de même depuis que les liposomes furent découverts et leur intérêt en tant que vecteur de principe actif ou tout simplement en tant qu'actif hydratant.

La suite présentera donc les deux aspects de ces molécules naturelles complexes c'est-à-dire leurs propriétés technologiques et physiologiques mais, comme on le verra, ces deux aspects sont bien souvent utilisés en même temps tant ils sont difficiles à dissocier.

\section{Les lipides polaires}

La figure 1 récapitule les grandes classes de lipides polaires que sont les phospholipides et glycolipides. Les phospholipides sont les composants essentiels des membranes cellulaires et sont donc présents dans tous les organismes vivants. La principale classe de phospholipides naturels est représentée par les glycérophospholipides qui dérivent du glycérol et dont la structure est reportée à la figure 2 ainsi que les principaux groupements polaires qui peuvent lui être associés.

Commercialement, les phospholipides sont principalement extraits du soja. Les phospholipides de soja contiennent plus de $70 \%$ d'acides gras mono- ou polyinsaturés mais des procédés d'hydrogénation permettent également d'obtenir des phospholipides aux acides gras saturés suivant le type d'utilisation.

La nature amphiphile des phospholipides leur permet d'agir comme coémulsifiants dans les systèmes eau/huile et huile/eau ainsi que comme épaississant, dispersant ou agent mouillant (figure 3).

Les phospholipides sont une source importante d'acides gras essentiels stables comme les acides linoléique et linolénique qui jouent un rôle essentiel au niveau de la fonction barrière de la peau (Prottey, 1977). Les phospholipides lient l'eau et leurs propriétés filmogènes contribuent à améliorer I'hydratation de la peau. Ce sont des substances non allergéniques, non irritan- tes qui permettent de diminuer l'irritation causée par les autres surfactants (Billek, 1991). La lécithine et les phospholipides qu'elle contient confèrent aux produits cosmétiques un toucher non gras, très agréable.

II existe une classe de phospholipides contenant une structure « sphingosine » permettant le classement dans les sphingolipides et que I'on trouve principalement dans les sources d'origine animale telle que le lait et l'œuf. Bien que peu utilisée aujourd'hui la sphingomyéline présente un potentiel important comme actif puisque son hydrolyse sur la peau se traduit par un apport de céramide aux acides gras très proches de ceux de la peau.

Moins connus, les glycolipides contiennent une partie polaire sucrée et l'on distingue de la même façon les glycéro- et sphingoglycolipides. Si les glycéroglycolipides sont préférentiellement rencontrés dans les plantes, les formes sphingosyl- sont présentes en quantités minoritaires comme par exemple les cérébrosides ou glycosylcéramide qui contiennent la précieuse molécule.

\section{Propriétés}

Ces phospholipides possèdent des propriétés structurelles permettant d'obtenir différentes organisations en phase aqueuse. Les formes «lyso- » ne contenant qu'une chaîne grasse forment préférentiellement des micelles alors que des structures telles que la phosphatidyl-

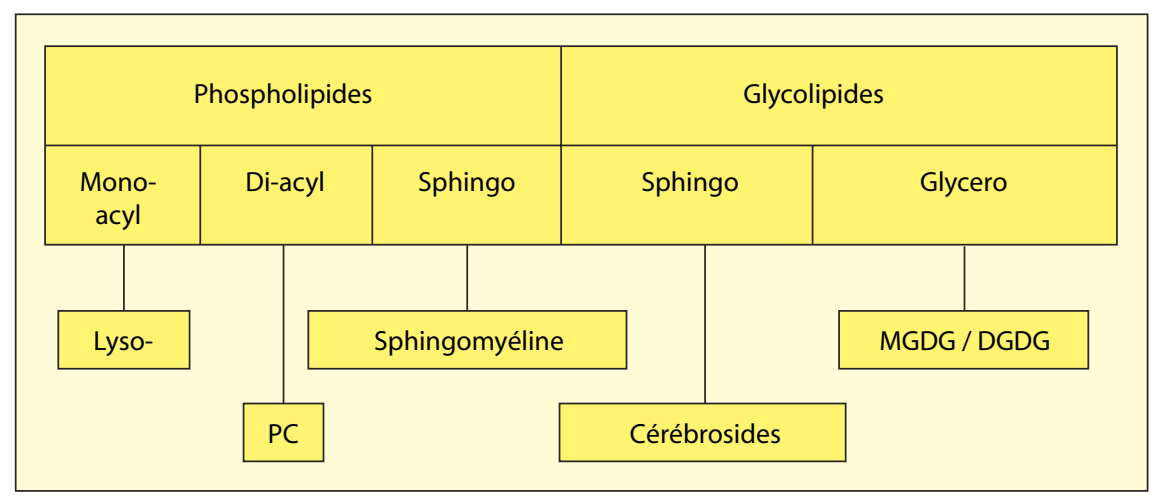

Figure 1. Lipides polaires (membranaires). 


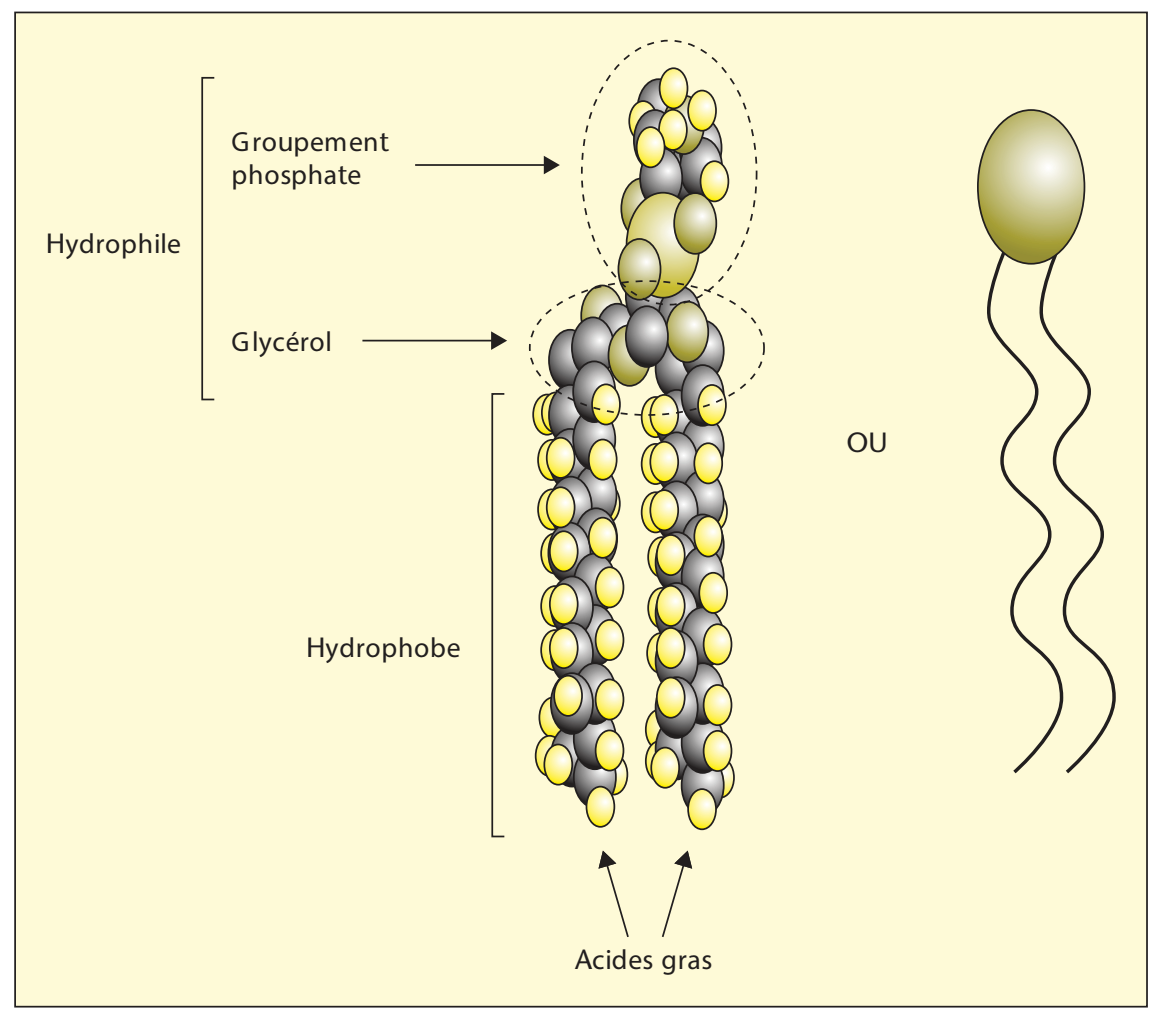

Figure 2. Structure des glycérophospholipides.

choline ou la sphingomyéline s'arrangent plutôt en bicouches se refermant sur elles-mêmes (liposomes). Une autre fraction, la phosphatidyléthanolamine, forme des structures lamellaires en présence de calcium.

Toutes ces phases permettent des applications différentes allant des très faibles teneurs en eau (micelles inverses) en passant par les émulsions lamellaires, les émulsions huile/eau et les liposomes pour ne citer que les plus connues.

Dans notre industrie, les premières applications furent réalisées en maquillage ou la liaison du groupement phosphate sur les pigments permet de les disperser dans la phase grasse tout en évitant leur agrégation. Cette propriété s'accompagne d'une baisse de la viscosité intéressante par exemple lors des coulages des rouges à lèvres.

De nombreuses préparations de soins huile/ eau utilisent comme émulsionnant principal ou secondaire une fraction de lécithine ou de phospholipides permettant de réduire ou d'éviter la présence d'émulsionnant de synthèse. Plusieurs études ont montré que ces émulsions sont généralement parfaitement tolérées et que des propriétés hydratantes apportent des revendications additionnelles.

Très souvent mis en avant, le toucher de ces formulations est un élément déterminant $d u$ choix de ce type d'émulsionnant. Frais, doux, non gras, sont les termes importants de l'analyse sensorielle de ce type d'émulsion.
La tête polaire constituée par le groupement phosphate des phospholipides, fait des lécithines de bons acteurs dans les systèmes antioxydants, ce qui explique que ces produits se conservent beaucoup plus longtemps à température ambiante que les huiles végétales.

D'autres propriétés pourraient encore être décrites mais la capacité de ces molécules à former des systèmes d'encapsulation est des plus intéressante. Comme précédemment décrit, des fractions bien choisies telles que la phosphatidylcholine forment des bicouches se reformant sur elles-mêmes qui vont pouvoir former des liposomes en présence d'eau. Ces réservoirs de l'ordre de $200 \mathrm{~nm}$ de diamètre peuvent encapsuler des actifs hydrophiles dans leurs espaces concentriques aqueux ou des molécules lipophiles dans leurs membranes. Particulièrement proche de la structure de la peau, ces membranes peuvent fusionner avec celle-ci et transporter les molécules actives plus profondément. Cette technique, qui n'est pas sans défaut, est une des seules qui permet d'utiliser des ingrédients naturels parfaitement tolérés.

\section{Émulsions lamellaires}

Le principal problème pour l'obtention de crèmes stables est d'éviter la coalescence des globules gras (crémage) ou la séparation des phases (rupture). La plupart des crèmes cosmétiques existantes utilisent le principe de monocouches à l'interface huile/eau et nécessitent donc I'utilisation de surfactants à hauts HLB irritants et/ou d'origine synthétique. Les surfactants à faibles HLB comme les acides ou alcools gras à longues chaînes sont incapables de for-

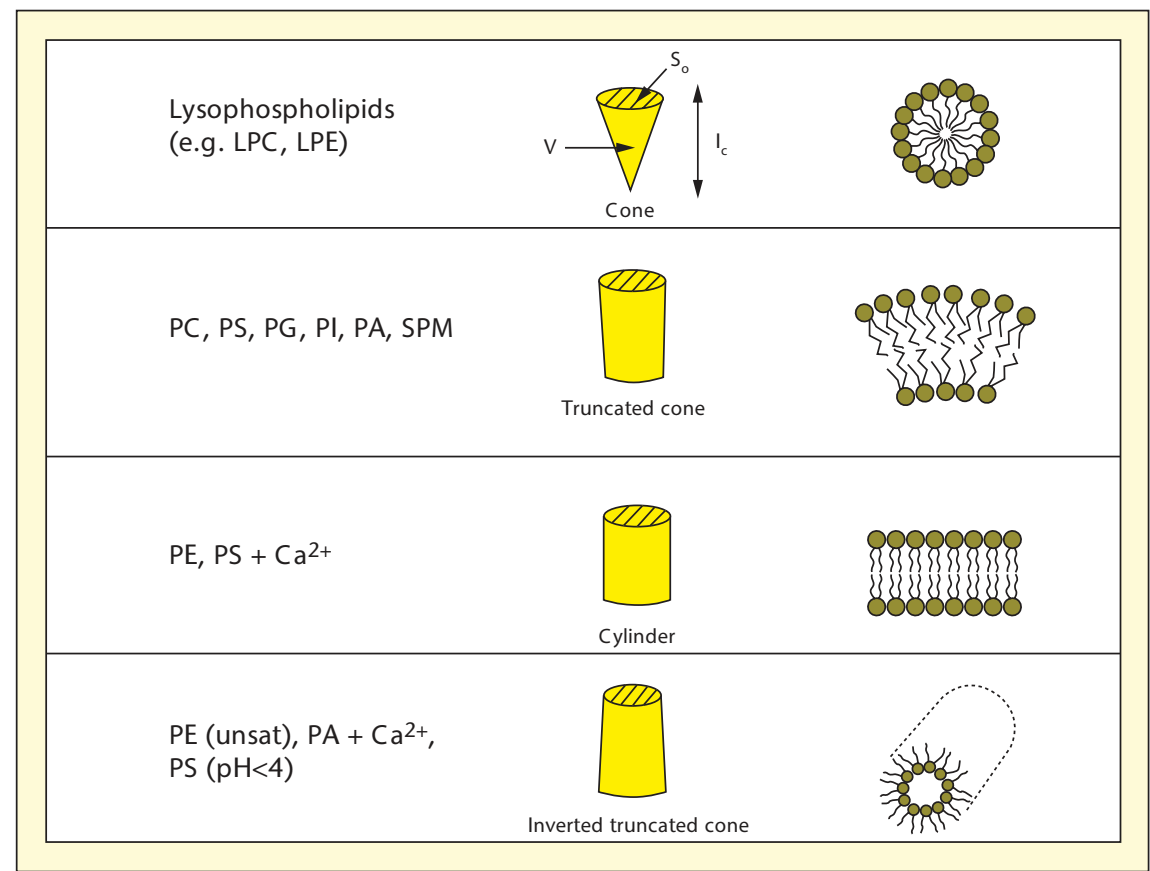

Figure 3. Structures formées par les différents types de phospholipides. 
mer des films de surface suffisamment résistants pour éviter la rupture des émulsions.

Les phospholipides sont également caractérisés par des faibles HLB et possèdent donc des propriétés émulsifiantes théoriques assez faibles. Leur combinaison avec certains acides et alcools gras permet cependant l'obtention de crèmes parfaitement stables jusqu'à $40 \%$ d'huile.

Ce nouveau type d'émulsifiant agit de deux manières. Initialement, les phospholipides se combinent aux autres composés à faibles HLB pour former des monocouches autour des gouttelettes de corps gras. Progressivement, un réseau de bicouches et de micelles mixtes de forte viscosité se forme dans la phase aqueuse qui isole les gouttelettes et confère au produit sa texture. Particulièrement proche de la structure de la peau, ces membranes peuvent fusionner avec celle-ci et transporter les molécules actives plus profondément.

Une représentation schématique de l'organisation structurelle de crèmes lamellaires à base de phospholipides est présentée figure 4. Les gouttelettes d'huile sont stabilisées à l'intérieur d'éléments complexes composés d'un mélange de phospholipides, d'acides et d'alcools gras. Le réseau lamellaire de telles structures dans la phase continue de l'émulsion, constitué $d^{\prime}$ un excès de composants renforce l'isolement des gouttes et prévient les phénomènes de crémage.

Une photographie en microscopie optique et électronique (cryofracture) sous forme hydratée en l'absence d'huile (formation d'un réseau gélifié) est présentée figure 4 et montre clairement la structure du réseau lamellaire.

Après 15 minutes d'application, un fin film de crème (et donc de phospholipides) peut clairement être observé contrairement au cas d'un épiderme non-traité.

La finesse de ce film a été estimée à environ 2 $\mu \mathrm{m}$. Sous un grossissement plus élevé, la structure de ce film peut être analysée. II est constitué d'une structure lamellaire (bicouche phospholipidique). Des vésicules couvrent la surface du modèle cutané fournissant une barrière lipidique et un réservoir de molécules d'eau et d'actifs encapsulés (figure 5).

Les résultats montrent que la perte insensible en eau a été sensiblement réduite comparativement au témoin (- $22 \%$ ) signifiant que les propriétés de barrières de la peau ont été restaurées grâce à l'émulsion lamellaire sur base phospholipidique.

\section{Propriétés physiologiques}

L'effet hydratant des lipides polaires ou plus exactement des phospholipides est souvent décrit dans la littérature et $s^{\prime}$ explique par deux raisons principales. Contenant des acides gras

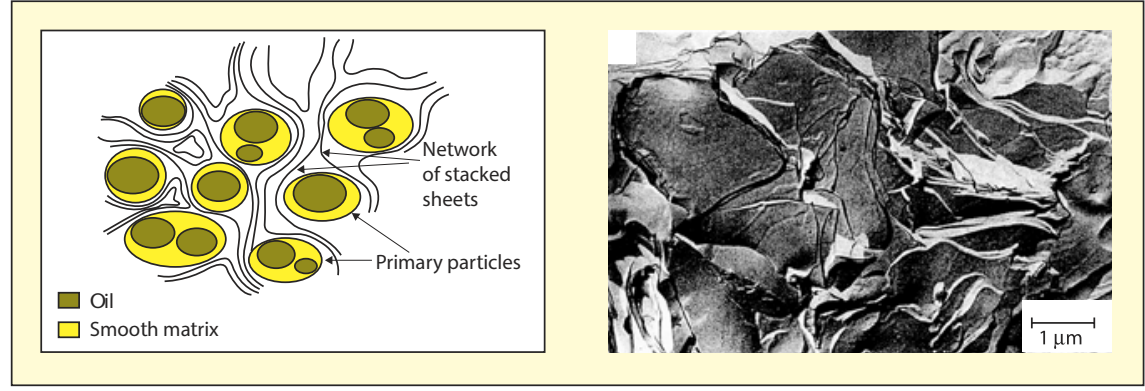

Figure 4. Représentation schématique de l'organisation structurelle de crèmes lamellaires à base de phospholipides.

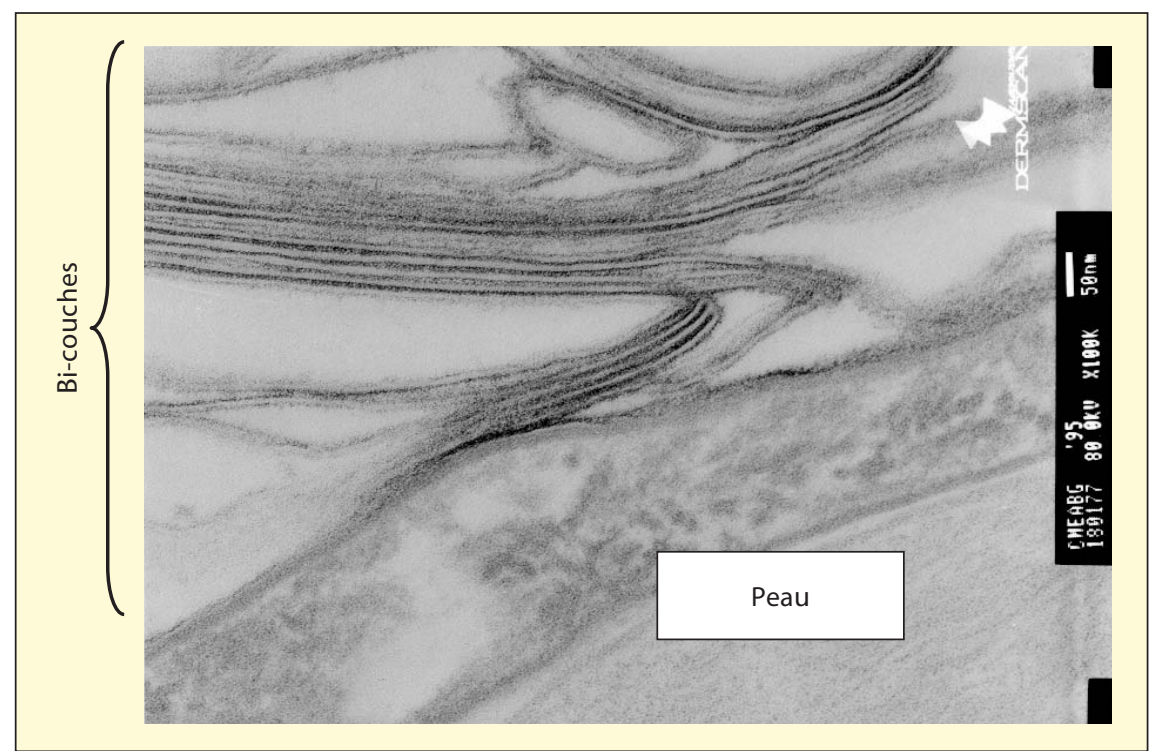

Figure 5. Des vésicules couvrent la surface du modèle cutané fournissant une barrière lipidique et un réservoir de molécules d'eau et d'actifs encapsulés.

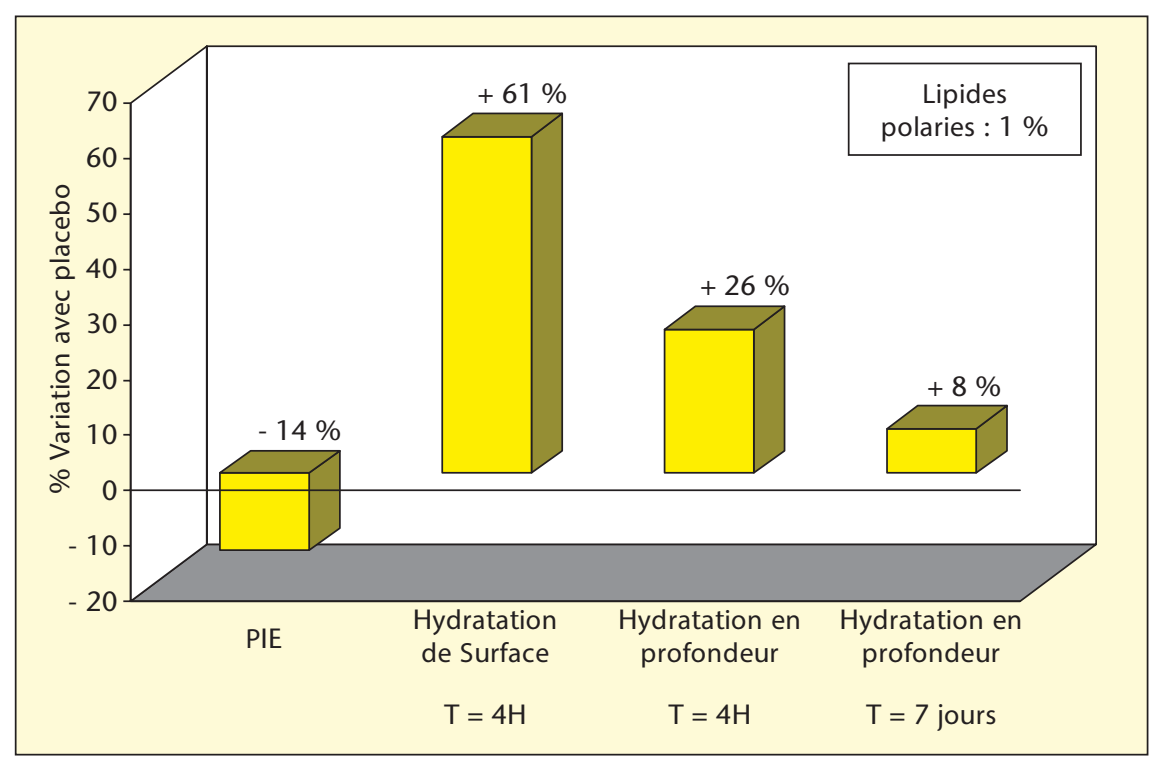

Figure 6. Une hydratation des couches superficielles et profondes importantes (+ $61 \%)$ quelques heures après application. 
essentiels (ou vitamine F), ces lipides procurent un effet hydratant à long terme. Grâce à leur propriété d'agrégation, les lécithines forment des réseaux lamellaires piégeant plus de dix fois leur poids en eau. Sans phospholipides, l'eau ne pourrait passer la barrière cutanée alors que prise dans cette structure membranaire, celle-ci pénètre plus facilement. Ainsi, plusieurs études ont montré un effet antidéshydratant ou comme sur la figure 6 une hydratation des couches superficielles et profondes importantes $(+61 \%)$ quelques heures après application. Les propriétés à long terme sont encore plus intéressantes puisqu'à 7 jours (soit 3 jours après le dernier traitement) une hydratation rémanente de $+8 \%$ est relevée.

L'effet anti-inflammatoire est très certainement dû également à la combinaison d'un effet physique de complexation des composés irritants tels que les tensio-actifs et d'un effet protecteur des acides gras polyinsaturés qui bloqueraient la cascade de l'acide arachidonique. Une réduction pratiquement totale de l'irritation a été mise en évidence lors de tests in vitro avec un composé irritant tel que le sodium dodecyl sulfate (voir figure 7).

Lors de tests in vitro sous UVB, une forte réduction de la synthèse des médiateurs de l'inflammation a été notée en présence de phospholipides allant jusquà réduire de $30 \%$ environ par rapport à un modèle de peau non traité, la concentration en prostaglandines E2 et interleukines 1alpha avec seulement $1 \%$ de lipides polaires.

Bien que moins décrites, les propriétés restructurantes de ces lipides cutanés sont bien réelles et de récentes études sur des lysolécithines (un acide gras ayant été enlevé par hydrolyse enzymatique) ont montré que celles-ci produisent une augmentation de l'expression des protéines de la jonction dermoépidermique telles que la laminine $\alpha-3$ et les collagènes IV et VII (figure 8). Cet effet restructurant in vitro a été vérifié sur volontaires et un effet lissant de $36 \%$ a été noté grâce à la présence des lipides.

\section{Aspects sécuritaires}

Puisque ces aspects prennent de plus en plus d'importance, il est important de noter que grâce à de nombreuses années d'utilisation, l'innocuité des lécithines et phospholipides a été largement prouvée. Utilisée comme additif alimentaire et autorisée par la FDA, la lécithine et les phospholipides ont fait l'objet d'un rapport du CIR Expert Panel en 1997 qui a conclu à l'innocuité des préparations en contenant jusqu'à $15 \%$ (!) aussi bien dans les produits rincés que non rincés. Non sensibilisant, non irritant pour la peau, très légèrement irritant pour les yeux, non mutagène, non photosensibilisant, etc. sont des points additionnels à mettre au profit de ces substances naturelles.

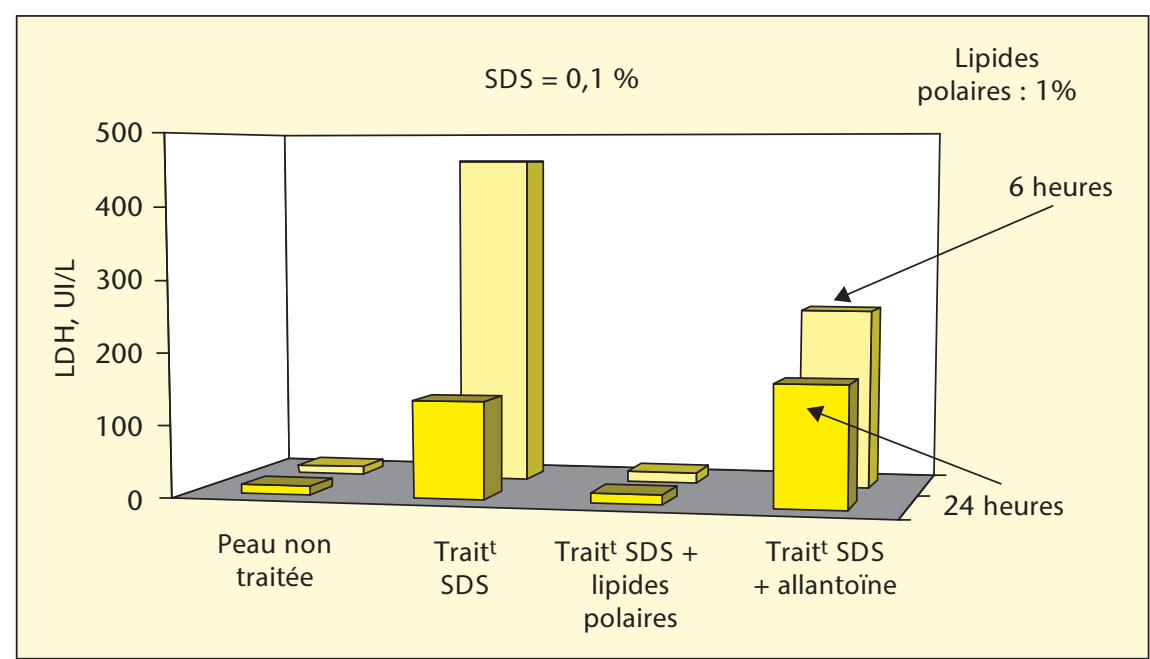

Figure 7. Une réduction pratiquement totale de l'irritation a été mise en évidence lors de tests in vitro avec un composé irritant tel que le sodium dodecyl sulfate.

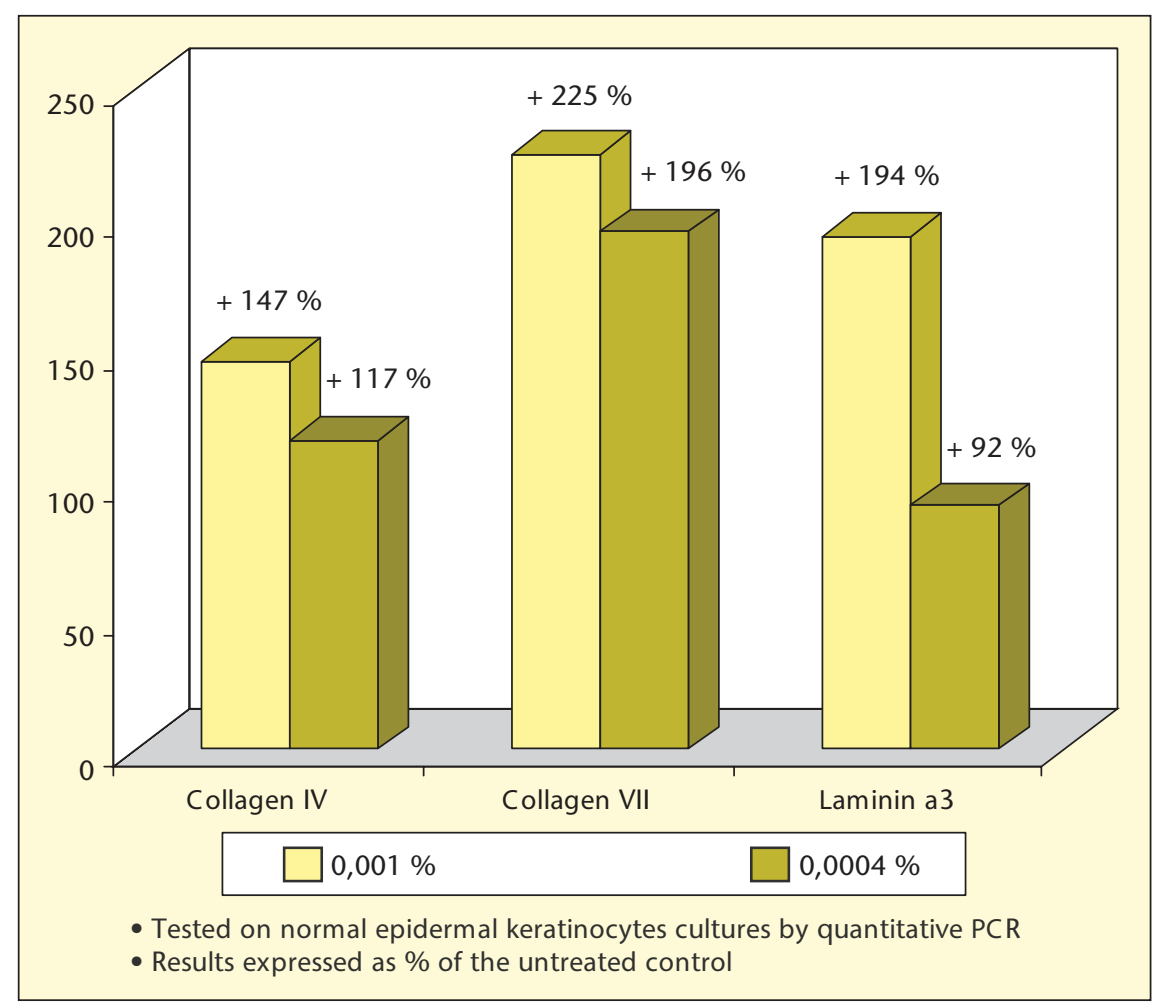

Figure 8. Expression des protéines de la jonction dermoépidermique.

\section{Conclusion}

Plus récemment, l'agrément Ecocert a été obtenu pour de nombreuses références contenant ces lipides membranaires et il est indiscutable que leur utilisation dans les préparations cosmétiques ne peut qu'augmenter pour suivre la tendance des consommateurs du « bien consommer ».
Pas toujours facile d'utilisation, la mise au point de produits en contenant réclame plus de temps mais les résultats tant sur le toucher que sur la tolérance et l'activité cosmétique sont indéniables. Pour reprendre une expression à la mode, ces lipides membranaires constituent une véritable seconde peau protégeant la nôtre tout en privilégiant le confort et le plaisir d'utilisation. 